\title{
Relation of Dietary Restraint Scores to Activation of Reward-Related Brain Regions in Response to Food Intake, Anticipated Intake, and Food Pictures
}

\author{
Kyle S. Burger \\ Eric Stice
}

Oregon Research Institute

Corresponding Author:

Kyle S. Burger

1715 Franklin Blvd. Eugene, OR 97403

Email: kyleb@ori.org

Phone: (541) 484-2123 x2169

Running Head: Dietary restraint \& activation of food reward-related brain regions Keywords: fMRI, Dietary restraint, OFC, DLPFC, Bulimia nervosa, Binge eating, Obesity, Dieting, Food pictures, anticipation and receipt, Food intake 


\begin{abstract}
Prospective studies indicate that individuals with elevated dietary restraint scores are at increased risk for future bulimic symptom onset, suggesting that these individuals may show hyper-responsivity of reward regions to food and food cues. Thus, we used functional magnetic resonance imaging (fMRI) to examine the relation of dietary restraint scores to activation of reward-related brain regions in response to receipt and anticipated receipt of chocolate milkshake and exposure to pictures of appetizing foods in 39 female adolescents (mean age $=15.5 \pm 0.94$ ). Dietary restraint scores were positively correlated with activation in the right orbitofrontal cortex (OFC) and bilateral dorsolateral prefrontal cortex (DLPFC) in response to milkshake receipt. However, dietary restraint scores did not correlate with activation in response to anticipated milkshake receipt or exposure to food pictures. Results indicate that individuals who report high dietary restraint have a hyper-responsivity in reward-related brain regions when food intake is occurring, which may increase risk for overeating and binge eating.
\end{abstract}




\section{Relation of Dietary Restraint Scores to Activation of Brain Reward Regions in Response to Food Intake, Anticipated Intake, and Food Pictures}

\section{Introduction}

Dieting, or dietary restraint, refers to intentional and sustained restriction of caloric intake for the purposes of weight loss or weight maintenance (Herman \& Mack, 1975; Wadden et al., 2002). Theorists posit that dieting increases risk for onset and maintenance of binge eating and bulimia nervosa (Fairburn, 1997; Levine \& Smolak, 2006; Neumark-Sztainer, 2005; Polivy \& Herman, 1985). Consistent with this theory, prospective risk factor studies indicate that females with high versus low scores on dietary restraint scales are at greater risk for future onset of binge eating, bulimic symptoms, and bulimic pathology (Killen et al., 1996; Neumark-Sztainer et al., 2006; Stice et al., 2008a; Stice et al., 1998) and increases in bulimic symptoms (Johnson \& Wardle, 2005; Stice, 2001; Wertheim et al., 2001). Because dieting is very prevalent, particularly among adolescent girls and young women (French et al., 1994; Serdula et al., 1993) and bulimic pathology is associated with functional impairment, distress, a chronic course, and elevated mortality risk (Grilo et al., 2003; Herzog et al., 2000; Stice et al., 2009), it is imperative to improve our understanding of the relation of dieting to bulimic pathology.

Surprisingly, studies using objective measures of caloric intake reveal that individuals with high versus low dietary restraint scores do not consume fewer calories and thus do not achieve the negative energy balance necessary for weight loss. Dietary restraint scores do not correlate inversely with unobtrusively observed caloric intake during single eating episodes (Epstein et al., 2004; Hetherington et al., 2000; Ouwens et al., 2003; Stice et al., 2004; Sysko et al., 2007) or multiple eating episodes (Jansen et al., 2003; Martin et al., 2005; Rolls et al., 1997; Sysko et al., 2005). Nor do high restraint scores correlate inversely with objective biological 
measures of habitual caloric intake over two week observational periods (Bathalon et al., 2000;

Stice et al., 2007; Stice et al., 2010a; Tuschl et al., 1990). In fact, there is evidence that individuals with high versus low dietary restraint scores show elevated weight (Laessle et al., 1989; Nederkoorn \& Jansen, 2001; Roefs et al., 2005) and increased risk for future weight gain (Klesges et al., 1992; Stice et al., 1999; Stice et al., 2005; Tanofsky-Kraff et al., 2007).

Emerging data support the thesis that obesity is associated with greater responsivity of reward circuitry to food and food cues. Functional magnetic resonance imaging (fMRI) studies show that obese versus lean individuals show greater activation in regions that encode the hedonic value and reward value of foods, including the insula, frontal operculum, parietal operculum, Rolandic operculum, lateral orbitofrontal cortex (OFC), amygdala, and striatum in response to pictures of palatable foods (Rothemund et al., 2007; Stoeckel et al., 2008; Stice et al., 2010b). Obese versus lean individuals also show greater activation in the gustatory cortex (frontal operculum) and somatosensory regions (parietal operculum) in response to consuming palatable food, which are regions that encode the sensory and hedonic aspects of food intake (Stice et al., 2008c). Yet, there is also evidence that obese relative to lean individuals show weaker activation in the dorsal striatum in response to receipt of palatable food (Stice et al., 2008b; Stice et al., 2008c), which appears to be a result of down-regulation resulting from overeating (Stice et al., 2010c).

There are evident parallels between characteristics of obese individuals and those that exhibit bulimic pathology. Obese versus lean individuals rate high-fat and high-sugar foods as more pleasant and consume more of such foods (McGloin et al., 2002; Nicklas et al., 2003; Rissanen et al., 2002) and work harder to earn food (Johnson, 1974; Saelens \& Epstein, 1996). Likewise, women with versus without bulimia nervosa typically rate sweet tastes as more 
pleasant, do not show normative decreases in pleasantness ratings of sweet tastes when consumed over time (Drewnowski et al., 1992; Franko et al., 1994; Rodin et al., 1990), and report greater urges to binge and less confidence in their ability to control their food intake after exposure to real food (Bulik et al., 1996; Staiger et al., 2000) but not after exposure to pictures of palatable foods (Bossert et al., 1991). Individuals with versus without bulimic pathology also rate pictures of food as more interesting and arousing and report a greater desire to eat, even when sated (Karhunen et al., 1997; Mauler et al., 2006). Participants with bulimia nervosa report greater urges to binge eat in response to exposure to both palatable and unpalatable foods (Staiger, et al., 2000), suggesting that they may anticipate greater reward from eating any food types. Bulimic pathology is also correlated with greater salivary response to food and less habituation of this response relative to controls (Bulik, et al., 1996; Vogele \& Florin, 1997; Wisniewski et al., 1997).

Similar findings have emerged from studies comparing those with elevated dietary restraint scores. Those with high versus low dietary restraint scores show greater salivary response to the sight and smell of real food (Klajner et al., 1981; Legenbauer et al., 2004; Legoff \& Spigelman, 1987; Sahakian et al., 1981) and greater cravings for palatable foods than those with lower scores (Gendall et al., 1998; Pelchat, 1997; Polivy et al., 2005), though null effects have also emerged (Nederkoorn \& Jansen, 2002; White et al., 2002). Individuals with elevated dietary restraint scores also report a greater likelihood to give in to food cravings and consume the craved food (Fedoroff et al., 1997; Polivy, et al., 2005). There is evidence that simply smelling food or thinking about eating food (Fedoroff, et al., 1997; Jansen \& van den Hout, 1991; Rogers \& Hill, 1989) leads to greater caloric intake among individuals with high but not low dietary restraint scores. However, individuals with high versus low dietary restraint scores 
did not differ in pleasantness ratings of palatable foods during taste tests or in how hard they work for food (Ahern et al., 2010; Fedoroff, et al., 1997; Goldfield \& Legg, 2006; Roefs, et al., 2005).

Although few studies have directly tested whether individuals with elevated dietary restraint scores exhibit hyper-responsivity of brain reward circuitry to food intake, a PET study found that dietary restraint scores were positively correlated with dopamine release in the dorsal striatum when exposed to the smell and taste of food (Volkow et al., 2003). An fMRI study found that individuals with high versus low dietary restraint scores showed greater activation in the OFC, dorsolateral prefrontal cortex (DLPFC) and insula in response to food pictures after consuming a meal, though participants did show similar activation patterns when calorically deprived (Coletta et al., 2009). Based on these findings it has been theorized that individuals with elevated dietary restraint scores may exhibit elevated responsivity of brain reward circuitry to food intake, anticipated intake, or food cues that increases risk for overeating and binge eating (Stice \& Presnell, 2010).

Collectively, the parallels in altered reward sensitivity between those who are overweight and those with bulimic pathology, and the positive relation between dietary restraint and onset of bulimic pathology suggest a positive relation between dietary restraint and reward sensitivity. To eliminate possible demand characteristics, we think it prudent to use objective brain imaging to test whether scores on dietary restraint scales correlate with activation of reward-related brain regions in response to intake of palatable foods. Thus, we investigated the relation between dietary restraint and reward-related neural responsivity to food intake, anticipated food intake, and pictures of food. We hypothesize that individuals reporting high dietary restraint scores 
would show hyper-responsivity in brain regions associated with food reward, as well as gustatory and somatosensory regions.

\section{Methods}

\subsection{Participants}

Participants were 39 healthy adolescent girls $(\mathrm{M}$ age $=15.5 \pm 0.94$, age range $=14-17$; $\mathrm{M}$ $\mathrm{BMI}=24.5 \pm 5.35, \mathrm{BMI}$ range $=17.3-38.9) ; 2 \%$ Asian $/$ Pacific Islanders, $2 \%$ African Americans, 86\% European Americans, 5\% Native Americans, and 5\% mixed racial heritage. Those who reported binge eating or compensatory behaviors in the previous three months, any use of psychotropic medications or illicit drugs, head injury with a loss of consciousness or current Axis I psychiatric disorder were excluded per Diagnostic and Statistical Manual of Mental Disorders, 4th edition criteria (American Psychiatric Assn, 1994). Informed consent was obtained from parents and assent from adolescents. The local Institutional Review Board approved this study.

\subsection{Procedures}

On the study day, participants were asked to consume their regular meals but to refrain from eating or drinking (including caffeinated beverages) for at least 4 - $6 \mathrm{hr}$ immediately preceding their imaging session for standardization purposes. This deprivation period was selected to capture the hunger state that most individuals experience as they approach their next meal, which is a time when individual differences in food reward would logically impact caloric intake. Although the participants were not observed during this time, participants reported a mean fasting time of $7.6 \pm 4.6$ hours prior to the scan time. No relations were observed between time fasting and restraint scores $(P=0.81)$. Participants completed two fMRI paradigms, which are described below and in prior reports (Stice et al., 2008b; Stice et al., 2010b,c), as well as a 
diagnostic screen, surveys, and measures of height and weight. Before the imaging session, participants were familiarized with the fMRI paradigms through practice on a separate computer.

Scanning was performed by a Siemens Allegra 3 Tesla head-only MRI scanner. A standard birdcage coil was used to acquire data from the entire brain. A thermo foam vacuum pillow and additional padding was used to restrict head motion. In total, 152 and 360 brain volumes were collected during each functional run for the consumption and anticipation paradigm and food picture exposure paradigm respectively. Functional scans used a T2weighted gradient single-shot echo planar imaging (EPI) sequence $(\mathrm{TE}=30 \mathrm{~ms}, \mathrm{TR}=2000 \mathrm{~ms}$, flip angle $\left.=80^{\circ}\right)$ with an in plane resolution of $3.0 \times 3.0 \mathrm{~mm} 2(64 \times 64$ matrix; $192 \times 192 \mathrm{~mm} 2$ field of view). To cover the whole brain, thirty-two 4-mm slices (interleaved acquisition, no skip) were acquired along the AC-PC transverse, oblique plane as determined by the midsagittal section. Structural scans were collected using an inversion recovery T1 weighted sequence (MP-RAGE) in the same orientation as the functional sequences to provide detailed anatomic images aligned to functional scans. High-resolution structural MRI sequences $(F O V=256 \times 256 \mathrm{~mm} 2,256 \times 256$ matrix, thickness $=1.0 \mathrm{~mm}$, slice number $\approx 160$ ) were also acquired.

\subsection{Consumption and Anticipation fMRI Paradigm}

The consumption and anticipation paradigm was designed to examine blood oxygen level dependent (BOLD) response when receiving or anticipating the receipt of a taste of a highly palatable food. Three black shapes (diamond, square, circle) were presented that signaled the impending delivery of either $0.5 \mathrm{ml}$ of a chocolate milkshake, a tasteless solution, or no delivery of fluid. Although the pairing of cues with stimuli and duration of stimulus presentation was randomly determined across participants, order of presentation was not randomized across participants. The tasteless solution was designed to mimic the natural taste of saliva (O'Doherty 
et al., 2001). On 50\% of the chocolate and tasteless solution trials, the taste was not delivered as expected to allow the investigation of the BOLD response to anticipation of a taste that was not confounded with actual receipt of the taste (unpaired trials). The events of interest in this paradigm were: (a) receipt of milkshake (milkshake receipt), (b) milkshake cue followed by no milkshake taste (unpaired milkshake cue), (c) receipt of tasteless solution (tasteless receipt), (d) tasteless solution cue followed by no tasteless solution (unpaired tasteless cue), and (e) no taste cue followed by no taste. The pictures were presented for 5 - $12 \mathrm{~s}(M=7)$ using MATLAB (Mathworks, Inc., Sherborn, MA). Taste delivery occurred 4 - $11 \mathrm{~s}(M=7)$ after onset of the cue. As a result, each event lasted between 4 and $12 \mathrm{~s}$. Each run consisted of four trials of each condition. Participants underwent four runs, resulting in 16 trials of each event. Tastes were delivered using programmable syringe pumps (Braintree Scientific BS-8000, Braintree, MA) operated through MATLAB to ensure consistent volume, rate, and timing of taste delivery. A manifold attached to the head coil fit into the participants' mouths and delivered the taste to a consistent tongue segment. The taste cue remained on the screen for $8.5 \mathrm{~s}$ after the taste was delivered, and participants were instructed to swallow when they saw the "swallow" cue. The next cue appeared 1-5 s after the prior cue went off. Pictures were presented with a digital projector/reverse screen display system to a screen at the back end of the MRI scanner bore and were visible via a mirror mounted on the head coil. These procedures have been successfully used in the past to deliver liquids in the scanner, differentiate between consumption and anticipation of consumption and have been described in detail elsewhere (Stice et al., 2008b,c; Veldhuizen et al., 2007).

2.4 Food Picture Exposure Paradigm 
This paradigm was designed to examine BOLD responses to exposure to and imagined intake of palatable foods, unpalatable foods, and glasses of water shown in pictures. Before the scanning session, participants rated how appetizing they found various foods shown in 103 pictures on a Lykert scale. The pictures presented to the participants included processed foods varied by type, energy density and macronutrient content (e.g., cheeseburger, cupcakes, crackers, cereal), fruits (e.g., grapes, peaches) and vegetables (e.g., cauliflower, eggplant). During the fMRI paradigm, each participant was exposed to the 20 pictures of food they rated as the most appetizing and the 20 pictures of food they rated as the least appetizing, as well as 20 pictures of glasses of water. Pictures were presented via the digital projector/reverse screen display system in 2 separate scanning runs. There were 3 events of interest in the paradigm: (1) viewing pictures of appetizing foods, (2) viewing pictures of unappetizing foods, and (3) viewing pictures of glasses of water. The pictures were presented for 5 seconds using Presentation (Version 9.81, www.neuro-bs.com). Order of presentation of the pictures was randomized. Each run consisted of 60 events ( 20 pictures each from the same category; appetizing, unappetizing, water). A blank screen with a cross-hair at the center for fixation (to eliminate random eye-movement) was presented for $2 \mathrm{~s}$ between each stimulus picture.

\subsection{Dutch Restrained Eating Scale.}

The Dutch Restrained Eating Scale (DRES; van Strien et al., 1986) assesses dietary behaviors designed to produce weight loss and weight maintenance (sample item: Do you deliberately eat less in order not to become too heavy?). This scale has shown internal consistency ( $\alpha$ 's range from .93 to .95 ) and temporal reliability; 2 -week test-retest $r=.82$ (Stice, et al., 2004; van Strien et al., 1986). Although this scale correlates with self-reported caloric 
intake (French, et al., 1994; Laessle, et al., 1989) it does not correlate with objectively measured caloric intake (Stice, et al., 2004; Stice, et al., 2010a).

\subsection{Imaging and statistical analysis}

All fMRI data were preprocessed and analyzed using SPM5 software (Wellcome Department of Imaging Neuroscience, London, England) in MATLAB (Mathworks, Inc., Sherborn, MA; Worsley \& Friston, 1995). The images were time acquisition corrected to the slice obtained at $50 \%$ of the TR. All images were motion corrected, realigned to the mean, normalized to the standard Montreal Neurological Institute (MNI) template brain implemented in SPM5 (ICBM152, based on an average of 152 MRI scans; voxel size of $3 \mathrm{~mm}^{3}$ for functional images and $1 \mathrm{~mm}^{3}$ for structural images). Data were spatially smoothed with a $6 \mathrm{~mm}$ full width at half maximum kernel and were high-pass filtered with a cutoff of $128 \mathrm{~s}$ to remove low frequency fluctuation in the BOLD signal.

Condition-specific effects at each voxel were estimated using general linear models. Vectors of the onsets for each event of interest were compiled and entered into the design matrix so that event-related responses could be modeled by the canonical hemodynamic response function (HRF), as implemented in SPM5, consisting of a mixture of two gamma functions that emulate the early peak at $5 \mathrm{~s}$ and the subsequent undershoot. To account for the variance induced by swallowing the solutions, the time of the "swallow" cue was included as a variable of no interest. Temporal derivatives of the HRF were also included to obtain a better model of the data (Henson et al., 2002).

To identify brain regions activated in response to receipt of a palatable food, BOLD response was contrasted during receipt of milkshake versus tasteless solution (milkshake receipt $>$ tasteless receipt). The arrival of a taste in the mouth was considered to be receipt. To identify 
brain regions activated in response to anticipated receipt, BOLD response during presentation of the cue signaling impending delivery of the milkshake was contrasted with response during presentation of the cue signaling impending delivery of the tasteless solution from unpaired trials (milkshake cue $>$ tasteless cue). In the food picture exposure paradigm contrasts of interest included BOLD responses while viewing pictures of appetizing food versus unappetizing food (appetizing food $>$ unappetizing food) and while viewing pictures appetizing food versus water (appetizing food $>$ water).

Individual SPM contrast maps were entered into regression models with dietary restraint scores with BMI as a covariate to test whether restraint scores correlated with BOLD response to consumption and anticipation of food and in response to viewing pictures of appetizing foods, controlling for the variance associated with BMI. We modeled dietary restraint scores as a continuous variable, rather than dichotomizing participants into high and low dietary restraint groups, as it should provide a more sensitive test of relations and it has been suggested that restraint is a continuous variable (Tomarken \& Kirschenbaum, 1984).

Small volume correction (SVC) was used for each of the contrasts of interest. Directed searches were performed in four regions of interest (ROIs) associated with: reward (caudate and putamen), encoding of reward (orbitofrontal cortex $[\mathrm{OFC}]$ and ventrolateral prefrontal cortex [VLPFC]), somatosensory cortex (rolandic operculum) and expectation of reward/behavioral control (dorsolateral prefrontal cortex [DLPFC]). The OFC was included as an ROI despite the possibility of signal dropout due to sinus and nasal cavities (Ojemann et al. 1997). The current slice angle was used to maximize signal coverage while minimizing Nyquest ghosts. Visual inspection of raw EPI was used to ensure adequate signal strength in this area (see Figure S1 for sample EPIs). ROIs were based on peaks from previous independent studies were used as 
centroids to define 10-mm diameter spheres (Coletta et al., 2009; DelParigi et al., 2007; Stice et al., 2008b; Stice et al., 2008c). To account for testing multiple regions ROIs were tested simultaneously using a single mask created in the WFU Pickatlas (Maldjian et al., 2003; Lancaster et al., 2000). Contrasts were set at a $T$-map threshold of $P<0.005$ with a minimum cluster of three. Activity in the selected areas of interest were considered significant with a $P<$ 0.05 corrected for multiple comparisons using the false discovery rate (FDR) technique (Genovese et al., 2002). Parameter estimates presented in figures were drawn from the specified peak voxel from the identified contrast using the above analytic techniques.

The likelihood of task (i.e., tasting) related motion was high in the current investigation and using motion parameters as nuance variables could statistically vary out effects of interest. As an alternative of using motion parameters as covariates, participants that showed excessive head movement (>2 mm) were excluded from that analysis. This was done to: 1) insure that the BOLD response to stimuli of interest were not statistically eliminated, 2) have the same threshold for both paradigms and 3) remain consistent with previous papers (Stice, et al., 2008b; Stice, et al., 2008c; Stice, et al., 2010b). In total, 10 of the 39 participants' BOLD data were not analyzed because they showed excessive head movement during the consumption and anticipation paradigm and 3 of the 39 participants' BOLD data were not analyzed because of excessive head movement during the picture exposure paradigm. Data is presented in mean \pm standard deviation unless otherwise noted.

\section{Results}

3.1 Relation of Dietary Restraint Scores to Consumption and Anticipated Consumption of Food $(n=29)$ 
Participants reported mean restraint scores of $22.5 \pm 9.4$ for the DRES (scale range 1050). DRES scores were regressed on activation in response to receipt of chocolate milkshake (versus tasteless solution) and activation in response to anticipated intake of chocolate milkshake (versus anticipated intake of tasteless solution), including BMI as a covariate.

In response to the milkshake receipt $>$ tasteless receipt, DRES scores were positively correlated with activation in the right OFC (MNI peaks 42, 51, -9; Figure 1A) and the bilateral DLPFC (-27, 48, 27 and 27, 48, 27; Figure 1B\&C). Effect sizes for these relations using parameter estimates from the peak voxels are graphical displayed in Figure 2A-C and are considered large (M semi-partial $r=0.54$; range 0.49- 0.57) using Cohen's criteria (Cohen, 1988). Additional information regarding these results can be seen in Table 1.

In response to anticipated receipt of chocolate milkshake (milkshake cue $>$ tasteless cue) DRES scores were not significantly related to with activation in the hypothesized regions.

\subsection{Relations of Dietary Restraint Scores to Pictures of Palatable Foods $(n=36)$}

DRES scores were regressed on activation in response viewing pictures of appetizing foods (versus glasses of water) and viewing pictures of appetizing foods (versus unappetizing foods) covaried with BMI. No significant relations were observed between regional brain activity in response to the picture exposure paradigm and restraint scores.

\section{Discussion}

In line with the hypothesis that individuals with high versus low dietary restraint scores would show hyper-responsivity from food stimuli in regions associated with food reward, OFC and DLPFC activation in response food receipt was positively associated with restraint scores. These results are in concert with reports of OFC and DLPFC activity in response to appetizing 
food pictures in individuals report high dietary restraint (Coletta et al., 2009). Consumption of a highly palatable food elicits activity in the OFC (O'Doherty et al., 2002; Small et al., 2008) and it has been proposed that this region plays a role in encoding food liking and other types of rewards (Kringelbach, 2005). Viewing high-calorie food has been found to activate the DLPFC, which has been implicated in expectation of reward and executive control (Aron et al., 2007; Killgore et al., 2003; Watanabe et al., 2002). This interpretation lines up with the notion that individuals with elevated dietary restraint scores may be hyper-responsive to the reward intake of palatable foods and may be attempting to modulate this response. Theorists have previously proposed a model of hyper-responsiveness of reward circuitry to food intake (Davis et al., 2004; Dawe \& Loxton, 2004). While this model is commonly associated with overeating and obesity, it is reasonable to suggest that individuals who report high dietary restraint have a similar a hyperresponsive reward circuitry that increases their risk for episodic overeating and binge eating.

When presented with appetizing food pictures and cues of impending food receipt, restraint scores were not significantly associated with activation in reward-related brain regions. There are clear differences between the consumption and anticipation paradigm and the picture exposure paradigm. For example, the consumption paradigm is more closely related to actual 'real world' food intake however, it presents only one food item. In contrast, the food picture exposure paradigm allows for the ability to vary the stimuli and use repeated presentations with no impact on satiety. It is possible that the inconsistency in the results between the responsivity to food receipt and anticipatory cues and food pictures could be restraint specific, as we have previously reported a relation between BMI and activity in reward-related regions in response to the picture exposure paradigm (Stice, et al., 2010b) and similar effects in response to the food receipt and anticipated receipt paradigm (Stice, et al., 2008c). Based on this, we hypothesize that 
individuals with high dietary restraint scores perceive food pictures as a less salient cue relative to those that involve actual food intake. This food consumption-mediated responsivity hypothesis for those that report high restraint is supported by the behavior literature. Specifically, women with bulimia nervosa typically rate sweet tastes higher and report greater urges to binge and less confidence in their ability to control their food intake after exposure to the sight, smell, and taste of real food compared to those without bulimia (Bulik, et al., 1996; Staiger, et al., 2000) but not after mere exposure to food pictures (Bossert, et al., 1991). Further, it has been suggested that those whom report high restraint may eat more food when fed because their appetite has been 'primed' by recent food intake and food subsequently appears more rewarding (Herman \& Polivy, 1984). This notion is supported by Coletta and colleagues (2009) reports of restraint related brain activity in the OFC and DLPFC when fed, but not fasted.

The current study has expanded on the work performed by Coletta and colleagues (2009) on several levels. In addition to using a food receipt paradigm, the current study used the Dutch restrained eating scale (as a continuous variable) compared dichotomizing the sample using on Herman and Polivy's Restraint Scale scores (Coletta, et al., 2009). Treating restraint as a continuous variable has been previously recommended (Tomarken \& Kirschenbaum, 1984) and doing so theoretically yields a more sensitive analysis. Parenthetically, when we tested whether the Three Factor Eating Questionnaire restraint scale (Stunkard \& Messick, 1985) correlated with activation in the paradigms examined in this study, similar results emerged (data not shown). Secondly, the method of picture selection used for the paradigm differed. Frequently fMRI studies have used picture exposure paradigms present predetermined pictures based on researchers' opinions of the hedonic value of the foods (e.g., Coletta, et al., 2009; Cornier et al., 2009) or pictures dichotomized based on caloric content (McCaffery et al., 2009; Rothemund, et 
al., 2007; Stoeckel, et al., 2008). In an effort to increase ecologically validity, this paradigm was designed to assess responsivity to foods participants themselves found appetizing. Despite the typical variability in food preferences from person to person, it was observed that participants rated foods in a similar manner, frequently rating dessert like foods and fruits high relative to vegetables, plain breakfast cereal, and crackers. Nevertheless, there is a small chance that this approach also could have introduced additional variance given that food pictures varied slightly from participant to participant. However, using the current picture exposure paradigm we have found similar effects reported by Stoeckel and colleagues (2008) and Rothemund and colleagues (2007) when relating regional brain reactivity to BMI (Stice, et al., 2010b), suggesting that if there is additional variance caused by this food selection method it is relatively small.

There are limitations present in the current study that should be noted. The current study used a slightly more liberal statistical $T$-map threshold $(P<0.005$ relative to $P<0.001)$ before correcting for multiple comparisons and multiple testing. Only the peak in the left DLPFC remained significant using the more stringent $T$-map threshold $(-27,48,27 ; k=3, p \mathrm{FDR}=$ 0.013). Secondly, the results could have been impacted because the order of the presentation of the paradigms was not randomized resulting in an order effect. Specifically, participants could have deduced that receipt of a palatable food was going to occur in a paradigm that took place after the picture exposure paradigm, thus altering the salience of the food pictures. Future studies comparing the salience of real food versus visual stimuli are required to clarify this. Additionally, the sample was adolescent females that reported no binge eating practices in the previous three months; therefore the results should be generalized to other populations with caution. The sample was drawn from a larger study, which was aimed to identify risk factors that predict the onset of bulimic pathology. The current study is relevant in that adolescent 
females are at a greater risk to develop bulimic pathology, for example binge eating (Lewinsohn et al., 1993). Finally, our ability to detect significant results could have been a function of lack of statistical power due to a small sample. However, it is of note that the current study has a comparative, if not larger, than sample sizes commonly seen in previous, similarly designed fMRI studies.

In sum, the present study has provided data to support that individuals with high dietary restraint scores show hyper-responsivity in brain regions that encode food reward during receipt of a palatable food, dovetailing with a previous brain imaging study (Coletta et al., 2009) and behavioral data. This possible heightened reward-related responsivity could increase the risk of binge eating. Additional prospective investigations using objective measures are needed to clarify the relations among self-reported dietary restraint, responsivity to a variety food stimuli, and susceptibility to the onset of bulimic pathology. 


\section{References}

Ahern, A., Field, M., Spoor, S., Bohon, C., \& Stice, E. (2010). Relation of dietary restraint scores to cognitive biases and reward sensitivity. Appetite, 55, 61-68.

American Psychiatric Assn. (1994). Diagnostic and statistical manual of mental disorders (4th ed.): Arlington, VA, US: American Psychiatric Publishing, Inc.

Aron, A. R., Behrens, T. E., Smith, S., Frank, M. J., \& Poldrack, R. A. (2007). Triangulating a cognitive control network using diffusion-weighted magnetic resonance imaging (MRI) and functional MRI. Journal of Neuroscience, 27(14), 3743-3752.

Bathalon, G. P., Tucker, K. L., Hays, N. P., Vinken, A. G., Greenberg, A. S., McCrory, M. A., et al. (2000). Psychological measures of eating behavior and the accuracy of 3 common dietary assessment methods in healthy postmenopausal women. American Journal of Clinical Nutrition, 71(3), 739-745.

Bossert, S., Laessle, R. G., Meiller, C., Junker, M., Ellgring, H., \& Pirke, K. M. (1991). Visual palatability of food in patients with eating disorders and dieting women. Behaviour Research and Therapy, 29(4), 337-341.

Bulik, C. M., Lawson, R. H., \& Carter, F. A. (1996). Salivary reactivity in restrained and unrestrained eaters and women with bulimia nervosa. Appetite, 27(1), 15-24.

Cohen, J. (1988). Statistical power analysis for the behavioral sciences (2nd ed.). Hisdale: Lawewnce Erlbaum Associates.

Coletta, M., Platek, S., Mohamed, F. B., van Steenburgh, J. J., Green, D., \& Lowe, M. R. (2009). Brain Activation in Restrained and Unrestrained Eaters: An fMRI Study. Journal of Abnormal Psychology, 118(3), 598-609. 
Cornier, M. A., Salzberg, A. K., Endly, D. C., Bessesen, D. H., Rojas, D. C., \& Tregellas, J. R. (2009). The Effects of Overfeeding on the Neuronal Response to Visual Food Cues in Thin and Reduced-Obese Individuals. PLoS ONE, 4(7), e6310.

Davis, C., Strachan, S., \& Berkson, M. (2004). Sensitivity to reward: implications for overeating and overweight. Appetite, 42(2), 131-138.

Dawe, S., \& Loxton, N. J. (2004). The role of impulsivity in the development of substance use and eating disorders. Neuroscience \& Biobehavioral Reviews, 28(3), 343-351.

DelParigi, A., Chen, K., Salbe, A. D., Hill, J. O., Wing, R. R., Reiman, E. M., et al. (2007). Successful dieters have increased neural activity in cortical areas involved in the control of behavior. International Journal of Obesity, 31(3), 440-448.

Drewnowski, A., Krahn, D. D., Demitrack, M. A., Nairn, K., \& Gosnell, B. A. (1992). Taste responses and preferences for sweet high-fat foods - evidence for opioid involvement. Physiology \& Behavior, 51(2), 371-379.

Epstein, L. H., Wright, S. M., Paluch, R. A., Leddy, J., Hawk, L. W., Jaroni, J. L., et al. (2004). Food hedonics and reinforcement as determinants of laboratory food intake in smokers. Physiology \& Behavior, 81(3), 511-517.

Fairburn, C. G. (1997). Eating disorders Science and practice of cognitive behaviour therapy (pp. 209-241): New York, NY, US: Oxford University Press.

Fedoroff, I. C., Polivy, J., \& Herman, C. P. (1997). The effect of pre-exposure to food cues on the eating behavior of restrained and unrestrained eaters. Appetite, 28(1), 33-47.

Franko, D. L., Wolfe, B. E., \& Jimerson, D. C. (1994). Elevated sweet taste pleasantness ratings in bulimia-nervosa. Physiology \& Behavior, 56(5), 969-973. 
French, S. A., Jeffery, R. W., \& Wing, R. R. (1994). Food intake and physical activity: a comparison of three measures of dieting. Addictive Behaviors, 19(4), 401-409.

Gendall, K. A., Joyce, P. R., Sullivan, P. F., \& Bulik, C. M. (1998). Personality and dimensions of dietary restraint. International Journal of Eating Disorders, 24(4), 371-379.

Genovese, C. R., Lazar, N. A., \& Nichols, T. (2002). Thresholding of statistical maps in functional neuroimaging using the false discovery rate. Neuroimage, 15(4), 870-878.

Goldfield, G. S., \& Legg, C. (2006). Dietary restraint, anxiety, and the relative reinforcing value of snack food in non-obese women. Eating Behaviors, 7(4), 323-332.

Grilo, C. M., Sanislow, C. A., Shea, M. T., Skodol, A. E., Stout, R. L., Pagano, M. E., et al. (2003). The natural course of bulimia nervosa and eating disorder not otherwise specified is not influenced by personality disorders. International Journal of Eating Disorders, 34(3), 319-330.

Henson, R. N., Price, C. J., Rugg, M. D., Turner, R., \& Friston, K. J. (2002). Detecting latency differences in event-related BOLD responses: application to words versus nonwords and initial versus repeated face presentations. Neuroimage, 15(1), 83-97.

Herman, C. P., \& Mack, D. (1975). Restrained and Unrestrained Eating. Journal of Personality, 43(4), 647-660.

Herman, C. P., \& Polivy, J. (1984). A boundary model for the regulation of eating. In A. Stunkard \& E. Stellar (Eds.), Eating and its disorders (pp. 141-156). New York: Raven Press.

Herzog, D. B., Greenwood, D. N., Dorer, D. J., Flores, A. T., Ekeblad, E. R., Richards, A., et al. (2000). Mortality in eating disorders: A descriptive study. International Journal of Eating Disorders, 28(1), 20-26. 
Hetherington, M. M., Stoner, S. A., Andersen, A. E., \& Rolls, B. J. (2000). Effects of acute food deprivation on eating behavior in eating disorders. International Journal of Eating Disorders, 28(3), 272-283.

Jansen, A., Theunissen, N., Slechten, K., Nederkoorn, C., Boon, B., Mulkens, S., et al. (2003). Overweight children overeat after exposure to food cues. Eating Behaviors, 4(2), $197-$ 209.

Jansen, A., \& van den Hout, M. (1991). On being led into temptation: "Counterregulation" of dieters after smelling a "preload". Addictive Behaviors, 16(5), 247-253.

Johnson, F., \& Wardle, J. (2005). Dietary restraint, body dissatisfaction, and psychological distress: a prospective analysis. Journal of Abnormal Psychology, 114(1), 119-125.

Johnson, W. G. (1974). Effect of cue prominence and subject weight on human food-directed performance. Journal of Personality and Social Psychology, 29(6), 843-848.

Karhunen, L. J., Lappalainen, R. I., Tammela, L., Turpeinen, A. K., \& Uusitupa, M. I. J. (1997). Subjective and physiological cephalic phase responses to food in obese binge-eating women. International Journal of Eating Disorders, 21(4), 321-328.

Killen, J. D., Taylor, C. B., Hayward, C., Haydel, K. F., Wilson, D. M., Hammer, L., et al. (1996). Weight concerns influence the development of eating disorders: a 4-year prospective study. Journal of Consulting and Clinical Psychology, 64(5), 936-940.

Killgore, W. D. S., Young, A. D., Femia, L. A., Bogorodzki, P., Rogowska, J., \& YurgelunTodd, D. A. (2003). Cortical and limbic activation during viewing of high- versus lowcalorie foods. Neuroimage, 19(4), 1381-1394.

Klajner, F., Herman, C. P., Polivy, J., \& Chhabra, R. (1981). Human obesity, dieting, and anticipatory salivation to food Physiology \& Behavior, 27(2), 195-198. 
Klesges, R. C., Isbell, T. R., \& Klesges, L. M. (1992). Relationship between dietary restraint, energy intake, physical activity, and body weight: a prospective analysis. Journal of Abnormal Psychology, 101(4), 668-674.

Kringelbach, M. L. (2005). The human orbitofrontal cortex: Linking reward to hedonic experience. Nature Reviews Neuroscience, 6(9), 691-702.

Laessle, R. G., Tuschl, R. J., Kotthaus, B. C., \& Pirke, K. M. (1989). A comparison of the validity of three scales for the assessment of dietary restraint. Journal of Abnormal Psychology, 98(4), 504-507.

Lancaster, J. L., Woldorff, M. G., Parsons, L. M., Liotti, M., Freitas, E. S., Rainey, L., et al. (2000). Automated Talairach Atlas labels for functional brain mapping. Human Brain Mapping, 10(3), 120-131.

Legenbauer, T., Vogele, C., \& Ruddel, H. (2004). Anticipatory effects of food exposure in women diagnosed with bulimia nervosa. Appetite, 42(1), 33-40.

Legoff, D. B., \& Spigelman, M. N. (1987). Salivary response to olfactory food stimuli as a function of dietary restraint and body-weight. Appetite, 8(1), 29-35.

Levine, M. P., \& Smolak, L. (2006). The prevention of eating problems and eating disorders: theory, research, and practice. Mahwah, NJ: Lawrence Earlbaum Associates.

Lewinsohn, P. M., Hops, H., Roberts, R. E., Seeley, J. R., \& Andrews, J. A. (1993). Adolescent psychopathology: I. Prevalence and incidence of depression and other DSM-III-R disorders in high school students. Journal of Abnormal Psychology, 102(1), 133-144.

Maldjian, J. A., Laurienti, P. J., Kraft, R. A., \& Burdette, J. H. (2003). An automated method for neuroanatomic and cytoarchitectonic atlas-based interrogation of fMRI data sets. Neuroimage, 19(3), 1233-1239. 
Martin, C. K., Williamson, D. A., Geiselman, P. J., Walden, H., Smeets, M., Morales, S., et al. (2005). Consistency of food intake over four eating sessions in the laboratory. Eating Behaviors, 6(4), 365-372.

Mauler, B. I., Hamm, A. O., Weike, A. I., \& Tuschen-Caffier, B. (2006). Affect regulation and food intake in bulimia nervosa: Emotional responding to food cues after deprivation and subsequent eating. Journal of Abnormal Psychology, 115(3), 567-579.

McCaffery, J. M., Haley, A. P., Sweet, L. H., Phelan, S., Raynor, H. A., Del Parigi, A., et al. (2009). Differential functional magnetic resonance imaging response to food pictures in successful weight-loss maintainers relative to normal-weight and obese controls. American Journal of Clinical Nutrition, 90(4), 928-934.

McGloin, A. F., Livingstone, M. B., Greene, L. C., Webb, S. E., Gibson, J. M., Jebb, S. A., et al. (2002). Energy and fat intake in obese and lean children at varying risk of obesity. International Journal of Obesity and Metabolic Disorders, 26(2), 200-207.

Nederkoorn, C., \& Jansen, A. (2001). Cue reactivity and eating behavior. Journal of Psychophysiology, 15(3), 216-216.

Nederkoorn, C., \& Jansen, A. (2002). Cue reactivity and regulation of food intake. Eating Behaviors, 3(1), 61-72.

Neumark-Sztainer, D. (2005). I'm, like, SO fat!: helping your teen make healthy choices about eating and excerise in a weight-obessed world. New York, NY: The Guilford Press.

Neumark-Sztainer, D., Wall, M., Guo, J., Story, M., Haines, J., \& Eisenberg, M. (2006). Obesity, disordered eating, and eating disorders in a longitudinal study of adolescents: how do dieters fare 5 years later? Journal of the American Dieteric Association, 106(4), 559-568. 
Nicklas, T. A., Yang, S. J., Baranowski, T., Zakeri, I., \& Berenson, G. (2003). Eating patterns and obesity in children. The Bogalusa Heart Study. American Journal of Preventive Medicine, 25(1), 9-16.

O'Doherty, J., Rolls, E. T., Francis, S., Bowtell, R., \& McGlone, F. (2001). Representation of pleasant and aversive taste in the human brain. Journal of Neurophysiology, 85(3), 13151321.

O'Doherty, J. P., Deichmann, R., Critchley, H. D., \& Dolan, R. J. (2002). Neural responses during anticipation of a primary taste reward. Neuron, 33(5), 815-826.

Ouwens, M. A., van Strien, T., \& van der Staak, C. P. F. (2003). Tendency toward overeating and restraint as predictors of food consumption. Appetite, 40(3), 291-298.

Pelchat, M. L. (1997). Food Cravings in Young and Elderly Adults. Appetite, 28(2), 103-113.

Polivy, J., Coleman, J., \& Herman, C. P. (2005). The effect of deprivation on food cravings and eating behavior in restrained and unrestrained eaters. International Journal of Eating Disorders, 38(4), 301-309.

Polivy, J., \& Herman, C. P. (1985). Dieting and binging. A causal analysis. American Psychologist, 40(2), 193-201.

Rissanen, A., Hakala, P., Lissner, L., Mattlar, C. E., Koskenvuo, M., \& Ronnemaa, T. (2002). Acquired preference especially for dietary fat and obesity: a study of weight-discordant monozygotic twin pairs. International Journal of Obesity, 26(7), 973-977.

Rodin, J., Bartoshuk, L., Peterson, C., \& Schank, D. (1990). Bulimia and taste - possible interactions. Journal of Abnormal Psychology, 99(1), 32-39. 
Roefs, A., Herman, C. P., MacLeod, C. M., Jansen, A., \& Smulders, F. T. Y. (2005). At first sight: How do restrained eaters evaluate high-fat palatable foods? Appetite, 44(1), 103114.

Rogers, P. J., \& Hill, A. J. (1989). Breakdown of dietary restraint following mere exposure to food stimuli - interrelationships between restraint, hunger, salivation, and food-intake Addictive Behaviors, 14(4), 387-397.

Rolls, B. J., Castellanos, V. H., Shide, D. J., Miller, D. L., Pelkman, C. L., Thorwart, M. L., et al. (1997). Sensory properties of a nonabsorbable fat substitute did not affect regulation of energy intake. American Journal of Clinical Nutrition, 65(5), 1375-1383.

Rothemund, Y., Preuschhof, C., Bohner, G., Bauknecht, H. C., Klingebiel, R., Flor, H., et al. (2007). Differential activation of the dorsal striatum by high-calorie visual food stimuli in obese individuals. Neuroimage, 37(2), 410-421.

Saelens, B. E., \& Epstein, L. H. (1996). Reinforcing value of food in obese and non-obese women. Appetite, 27(1), 41-50.

Sahakian, B. J., Lean, M. E. J., Robbins, T. W., \& James, W. P. T. (1981). Salivation and insulin-secretion in response to food in nonobese men and women. Appetite, 2(3), 209216.

Serdula, M. K., Collins, M. E., Williamson, D. F., Anda, R. F., Pamuk, E., \& Byter, T. E. (1993). Weight control practices of United-States adolescents and adults. Annals of Internal Medicine, 119(7), 667-671.

Small, D. M., Veldhuizen, M. G., Felsted, J., Mak, Y. E., \& McGlone, F. (2008). Separable substrates for anticipatory and consummatory food chemosensation. Neuron, 57(5), 786797. 
Staiger, P., Dawe, S., \& McCarthy, R. (2000). Responsivity to food cues in bulimic women and controls. Appetite, 35(1), 27-33.

Stice, E. (2001). A prospective test of the dual-pathway model of bulimic pathology: mediating effects of dieting and negative affect. Journal of Abnormal Psychology, 110(1), 124-135.

Stice, E., Cameron, R. P., Killen, J. D., Hayward, C., \& Taylor, C. B. (1999). Naturalistic weight-reduction efforts prospectively predict growth in relative weight and onset of obesity among female adolescents. Journal of Consulting and Clinical Psychology, 67(6), 967-974.

Stice, E., Cooper, J. A., Schoeller, D. A., Tappe, K., \& Lowe, M. R. (2007). Are dietary restraint scales valid measures of moderate- to long-term dietary restriction? Objective biological and behavioral data suggest not. Psychological Assessment, 19(4), 449-458.

Stice, E., Davis, K., Miller, N. P., \& Marti, C. N. (2008a). Fasting increases risk for onset of binge eating and bulimic pathology: a 5-year prospective study. Journal of Abnormal Psychology, 117(4), 941-946.

Stice, E., Fisher, M., \& Lowe, M. R. (2004). Are dietary restraint scales valid measures of acute dietary restriction? Unobtrusive observational data suggest not. Psychological Assessment, 16(1), 51-59.

Stice, E., Killen, J. D., Hayward, C., \& Taylor, C. B. (1998). Age of onset for binge eating and purging during late adolescence: a 4-year survival analysis. Journal of Abnormal Psychology, 107(4), 671-675.

Stice, E., Marti, C. N., Shaw, H., \& Jaconis, M. (2009). An 8-year longitudinal study of the natural history of threshold, subthreshold, and partial eating disorders from a community sample of adolescents. Journal of Abnormal Psychology, 118(3), 587-597. 
Stice, E., \& Presnell, K. (2010). Dieting and the Eating Disorders The Oxford Handbook of Eating Disorders. New York, NY: Oxford University Press.

Stice, E., Presnell, K., Shaw, H., \& Rohde, P. (2005). Psychological and behavioral risk factors for obesity onset in adolescent girls: a prospective study. Journal of Consulting and Clinical Psychology, 73(2), 195-202.

Stice, E., Spoor, S., Bohon, C., \& Small, D. M. (2008b). Relation between obesity and blunted striatal response to food is moderated by TaqIA A1 allele. Science, 322(5900), 449-452.

Stice, E., Spoor, S., Bohon, C., Veldhuizen, M. G., \& Small, D. M. (2008c). Relation of Reward From Food Intake and Anticipated Food Intake to Obesity: A Functional Magnetic Resonance Imaging Study. Journal of Abnormal Psychology, 117(4), 924-935.

Stice, E., Sysko, R., Roberto, C. A., \& Allison, S. (2010a). Are dietary restraint scales valid measures of dietary restriction? Additional objective behavioral and biological data suggest not. Appetite, 54(2), 331-339.

Stice, E., Yokum, S., Blum, K., \& Bohon, C. (2010c). Weight gain associated with reduced striatal response to palatable food. Journal of Neruoscience, 30, 13105-13109.

Stice, E., Yokum, S., Bohon, C., Marti, N., \& Smolen, A. (2010b). Reward circuitry responsivity to food predicts future increases in body mass: Moderating effects of DRD2 and DRD4. Neuroimage, 50(4), 1618-1625.

Stoeckel, L. E., Weller, R. E., Cook, E. W., Twieg, D. B., Knowlton, R. C., \& Cox, J. E. (2008). Widespread reward-system activation in obese women in response to pictures of highcalorie foods. Neuroimage, 41(2), 636-647.

Stunkard, A., \& Messick, S. (1985). The Three Factor Eating Questionnaire to Measure Dietary Restraint, Disinhibition, and Hunger. Journal of Psychosomatic Research, 29 (1), 71-83. 
Sysko, R., Walsh, B. T., Schebendach, J., \& Wilson, G. T. (2005). Eating behavior among women with anorexia nervosa. American Journal of Clinical Nutrition, 82(2), 296-301.

Sysko, R., Walsh, B. T., \& Wilson, G. T. (2007). Expectancies, dietary restraint, and test meal intake among undergraduate women. Appetite, 49(1), 30-37.

Tanofsky-Kraff, M., Wilfley, D. E., Young, J. F., Mufson, L., Yanovski, S. Z., Glasofer, D. R., et al. (2007). Preventing excessive weight gain in adolescents: Interpersonal psychotherapy for binge eating. Obesity, 15(6), 1345-1355.

Tomarken, A. J., \& Kirschenbaum, D. S. (1984). Effects of plans for future meals on counterregulatory eating by restrained and unrestrained eaters. Journal of Abnormal Psychology, 93(4), 458-472.

Tuschl, R. J., Platte, P., Laessle, R. G., Stichler, W., \& Pirke, K. M. (1990). Energy-expenditure and everyday eating behavior in healthy-young women. American Journal of Clinical Nutrition, 52(1), 81-86.

van Strien, T., Frijters, J. E., Van Staveren, W. A., \& Defares, P. B. (1986). The predictive validity of the Dutch Restrained Eating Scale. International Journal of Eating Disorders, 5(4), 747-755.

Veldhuizen, M. G., Bender, G., Constable, R. T., \& Small, D. M. (2007). Trying to detect taste in a tasteless solution: modulation of early gustatory cortex by attention to taste. Chemical Senses, 32(6), 569-581.

Vogele, C., \& Florin, I. (1997). Psychophysiological responses to food exposure: An experimental study in binge eaters. International Journal of Eating Disorders, 21(2), $147-157$. 
Volkow, N. D., Wang, G. J., Maynard, L., Jayne, M., Fowler, J. S., Zhu, W., et al. (2003). Brain dopamine is associated with eating behaviors in humans. International Journal of Eating Disorders, 33(2), 136-142.

Wadden, T. A., Brownell, K. D., \& Foster, G. D. (2002). Obesity: Responding to the global epidemic. Journal of Consulting and Clinical Psychology, 70(3), 510-525.

Watanabe, J., Sugiura, M., Sato, K., Sato, Y., Maeda, Y., Matsue, Y., et al. (2002). The human prefrontal and parietal association cortices are involved in NO-GO performances: An event-related fMRI study. Neuroimage, 17(3), 1207-1216.

Wertheim, E. H., Koerner, J., \& Paxton, S. J. (2001). Longitudinal predictors of restrictive eating and bulimic tendencies in three different age groups of adolescent girls. Journal of Youth and Adolescence, 30(1), 69-81.

White, M. A., Whisenhunt, B. L., Williamson, D. A., Greenway, F. L., \& Netemeyer, R. G. (2002). Development and validation of the food-craving inventory. Obesity Research, $10(2), 107-114$.

Wisniewski, L., Epstein, L. H., Marcus, M. D., \& Kaye, W. (1997). Differences in salivary habituation to palatable foods in bulimia nervosa patients and controls. Psychosomatic Medicine, 59(4), 427-433.

Worsley, K. J., \& Friston, K. J. (1995). Analysis of fMRI time-series revisited--again. Neuroimage, 2(3), 173-181. 


\section{Acknowledgements}

Support for this work was provided by National Institute of Health Research Grant R1MH64560. The authors would like to thank Sonja Yokum and Janet $\mathrm{Ng}$ for their assistance in data collection and analysis. The authors declare no conflict of interest. 
Table 1.

Increases in regional brain activity in response to consumption of food as a function of dietary restraint.

\begin{tabular}{lccccc}
\hline & $\begin{array}{c}\text { Reference } \\
\text { coordinates }^{\mathrm{a}}\end{array}$ & $x, y, z^{b}$ & $k$ & $Z$ value & $\begin{array}{c}p \text { FDR } \\
\text { corrected }^{c}\end{array}$ \\
\hline Obitofrontal cortex (OFC) & $43,49,-16$ & $42,51,-9$ & 4 & 3.05 & 0.036 \\
$\begin{array}{l}\text { Dorsolateral prefrontal } \\
\text { cortex (DLPFC) }\end{array}$ & $33,51,30$ & $27,48,27$ & 6 & 2.95 & 0.036 \\
& & $-27,48,27$ & 12 & 3.47 & 0.036 \\
\hline
\end{tabular}

\footnotetext{
${ }^{a}$ Reference coordinates from DelParigi et al. (2007) and Coletta et al. (2009)

${ }^{\mathrm{b}}$ Stereotactic coordinates in MNI space (Internet: http://mni.mcgill.ca/).

${ }^{\mathrm{c}} T$-maps thresholded at $P<0.005$ (uncorrected) with a cluster threshold of three. Peaks were considered significant $P<0.05$ using false-discovery rate (FDR) correction for multiple comparisons.
} 


\section{Figures}

Figure 1. Increases in activity in the A) right orbtiofrontal cortex (OFC; MNI peak 42, 51, -9) and the B) right $(27,48,27)$ and $\mathbf{C})$ left $(-27,48,27)$ dorsolateral prefrontal cortex (DLPFC) in response to the milkshake receipt $>$ tasteless receipt as a function of dietary restraint as measured by the Dutch restrained eating questionnaire covaired with BMI.

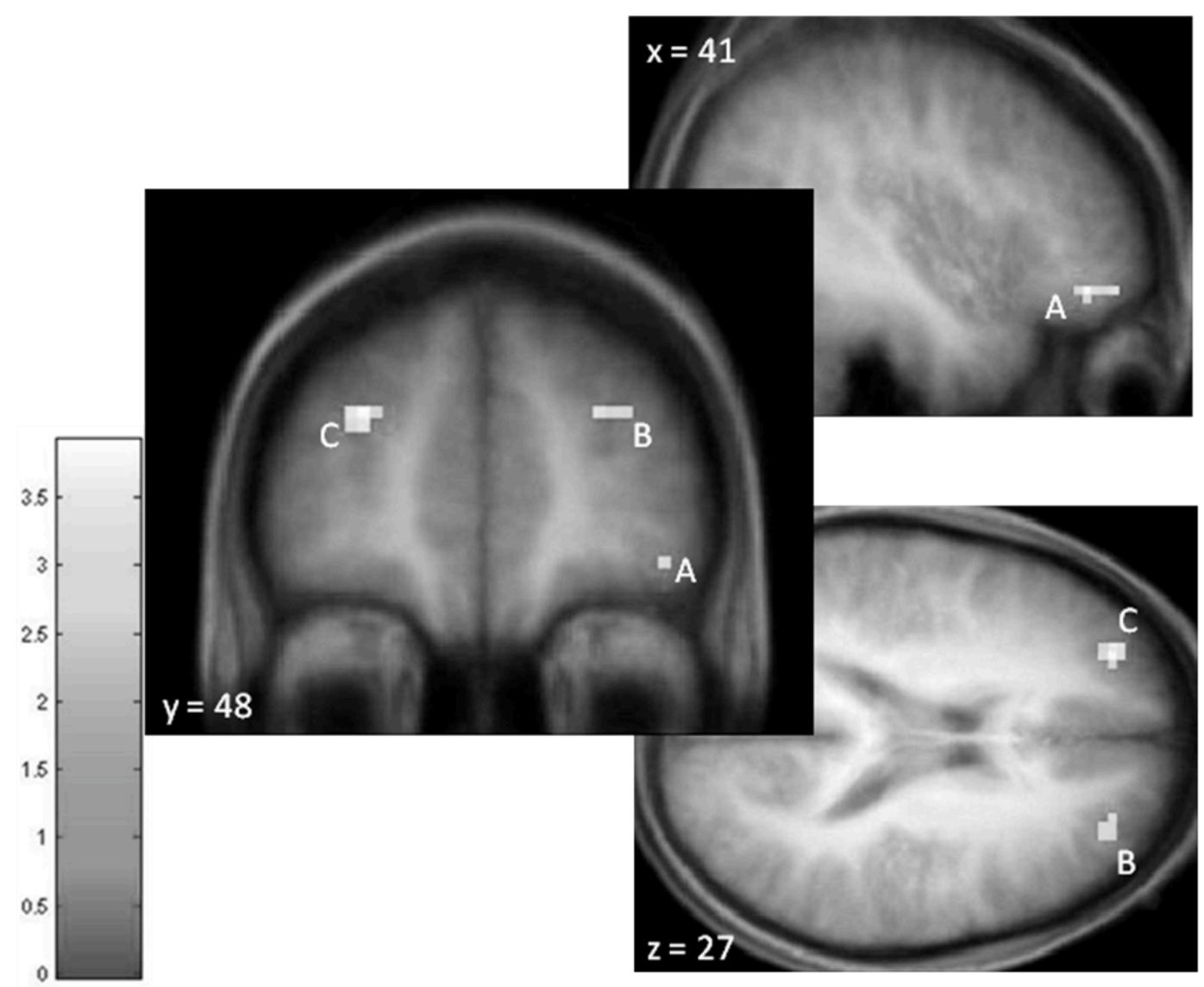


Figure 2. Parameter estimates from activation in the peak voxel observed in the $\mathbf{A}$ ) right orbtiofrontal cortex (OFC; MNI peak 42, 51, -9) and the B) right $(27,48,27)$ and C) left (-27, 48,27 ) dorsolateral prefrontal cortex (DLPFC) in response to the milkshake receipt $>$ tasteless receipt as a function of dietary restraint as measured by the Dutch restrained eating questionnaire covaired with BMI.

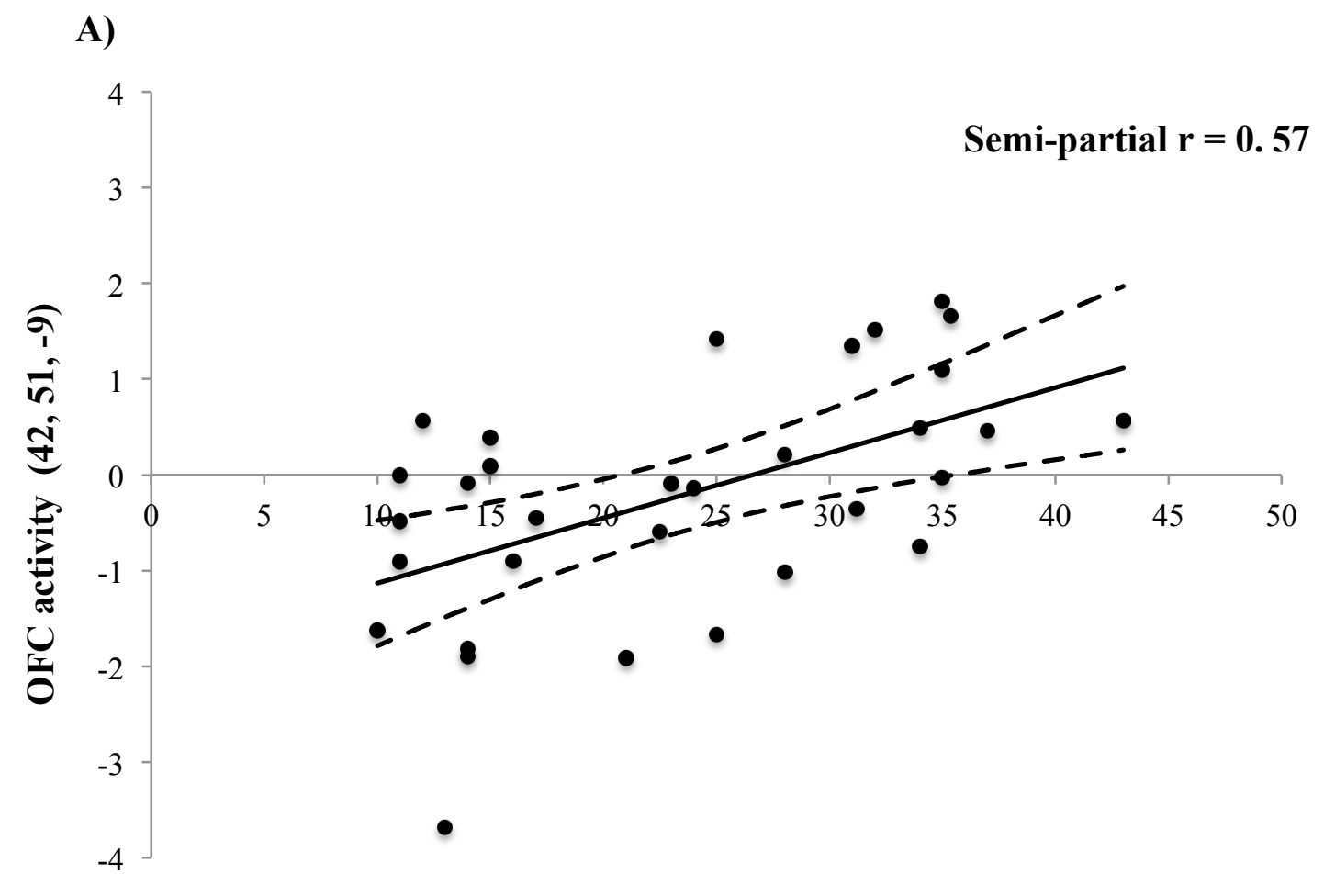

Restraint scores 
B)

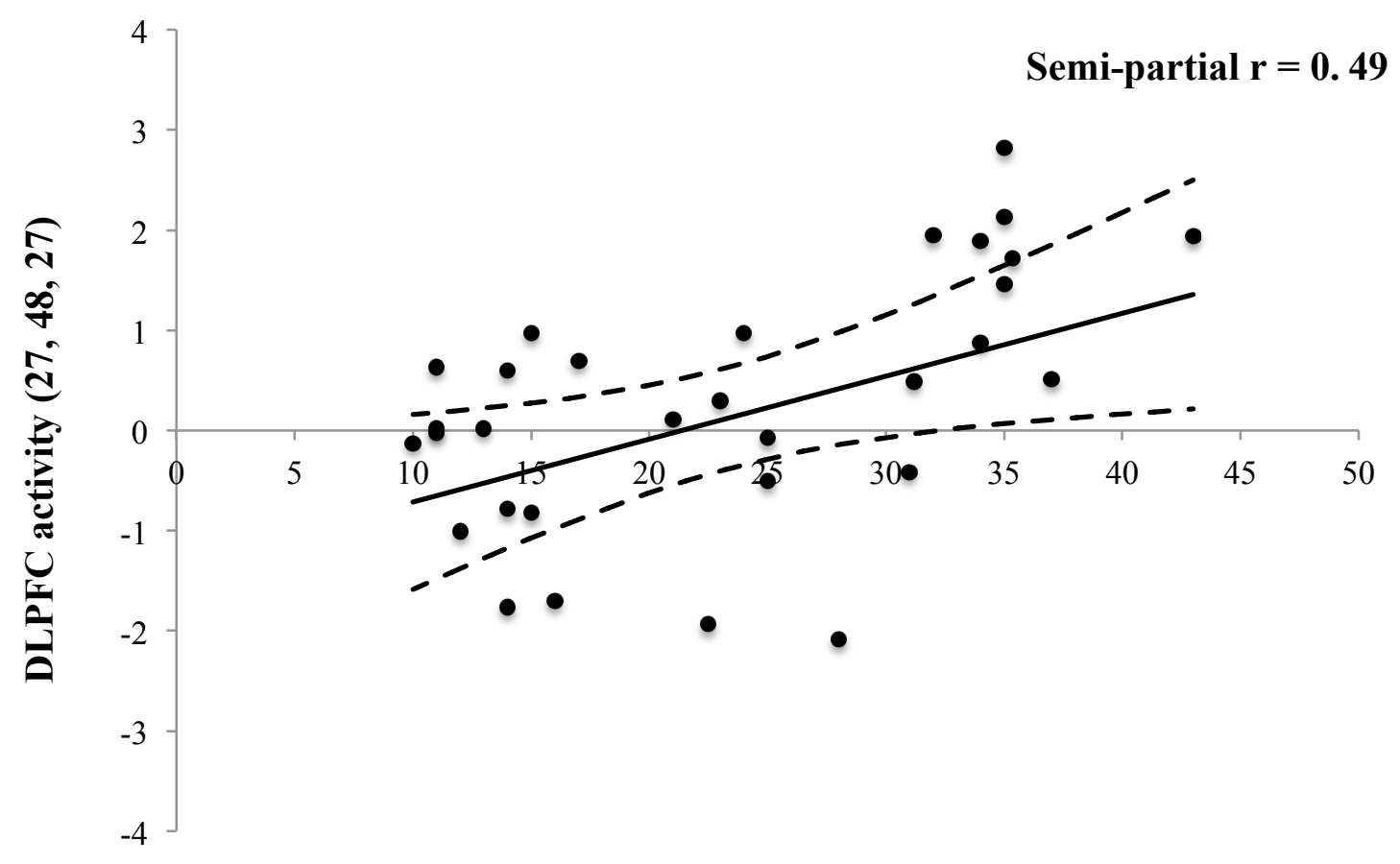

Restraint scores

C)

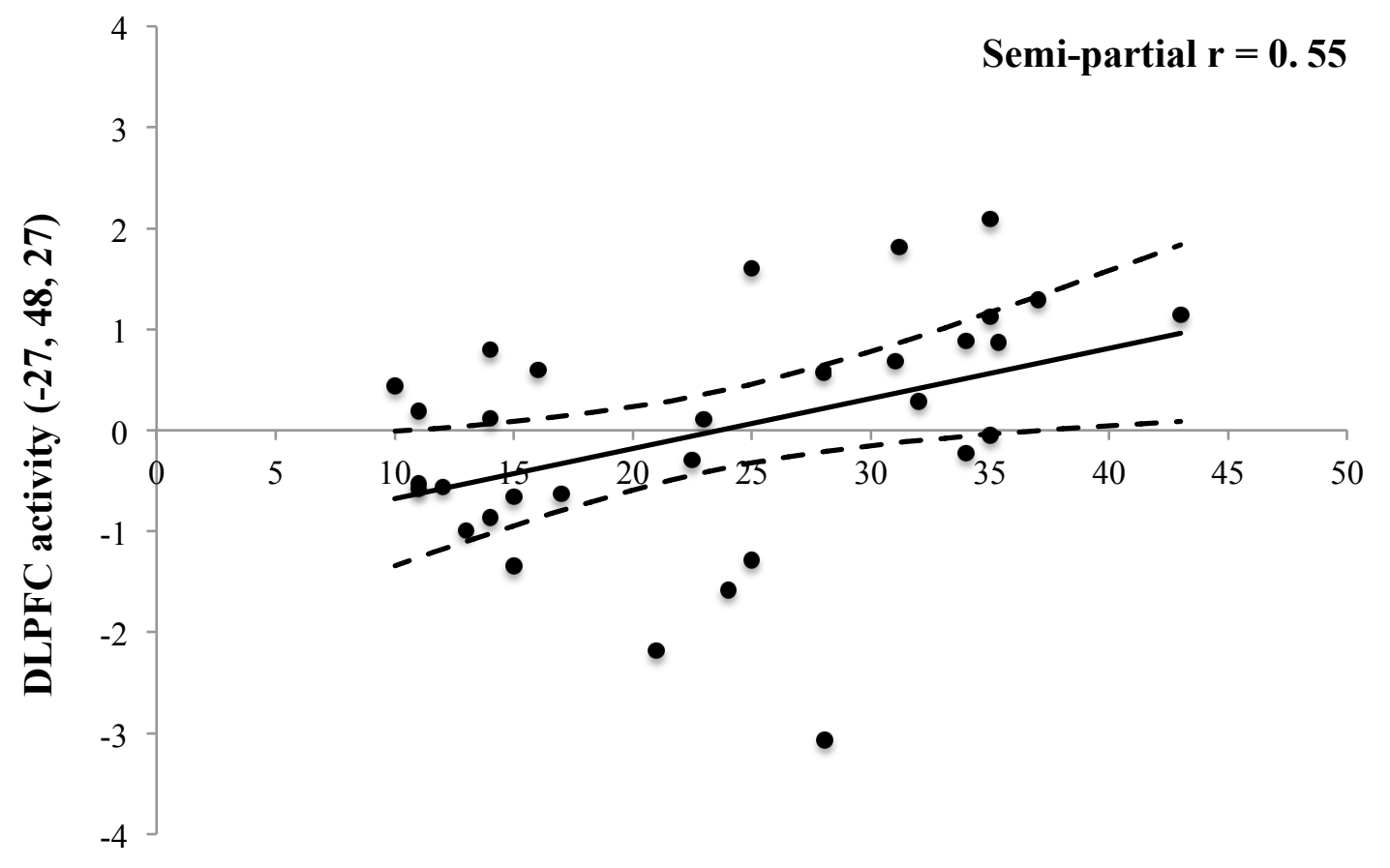

Restraint scores 


\section{Figure S1.}

Sample EPI images from four participants while performing the consumption and anticipation paradigm demonstrating adequate signal coverage in the area of the orbitofrontal cortex (MNI coordinate $42,51,-9)$.
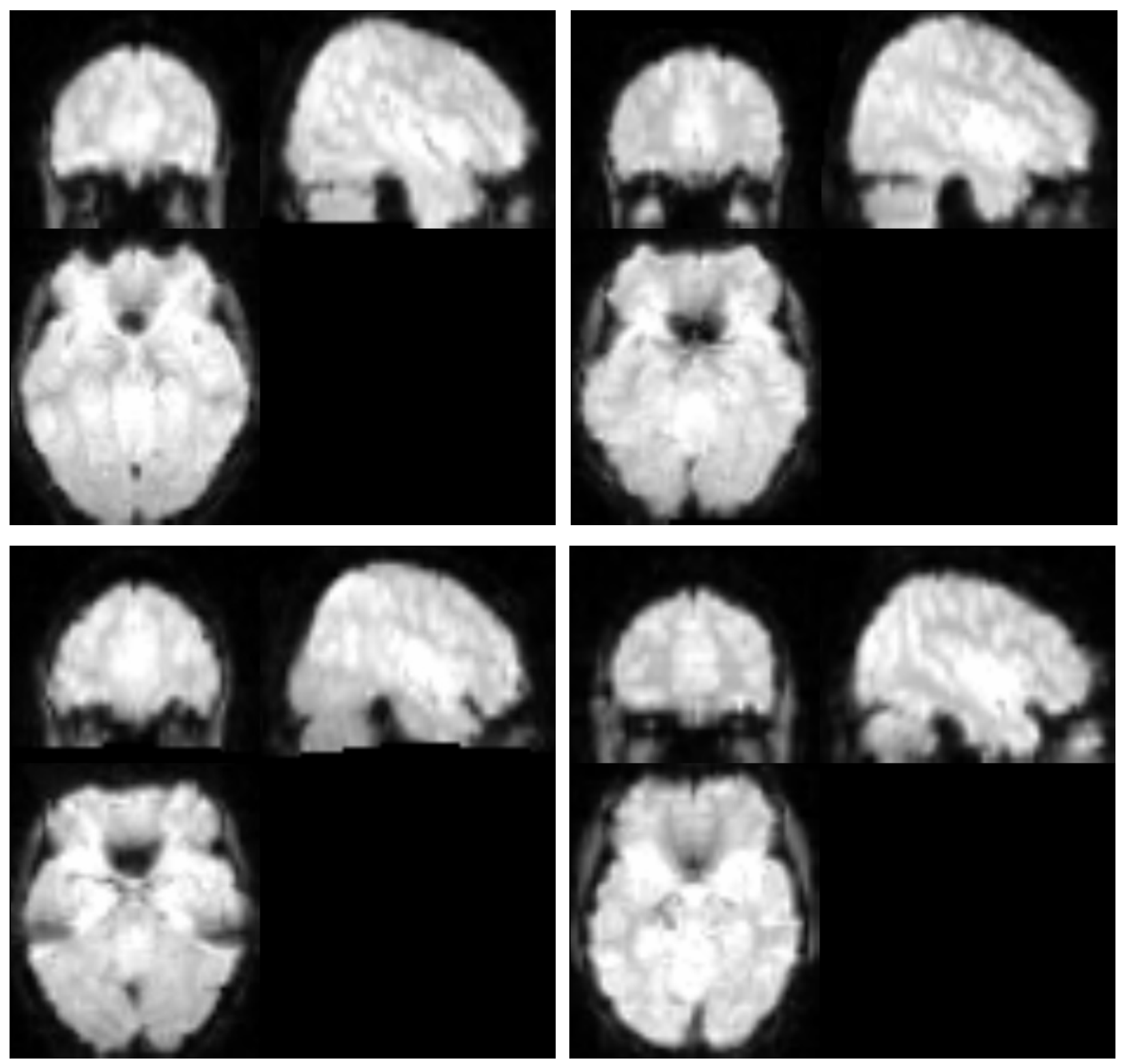